\title{
On the Convergence of the $p$-Version of the Boundary Element Galerkin Method
}

\author{
By E. P. Stephan and M. Suri
}

\begin{abstract}
We prove convergence for the $p$-version of Galerkin boundary element schemes applied to various first-kind integral equations. We establish optimal error estimates for the $p$-version in the $H^{1 / 2}$ and $H^{-1 / 2}$-norms and also derive rates of convergence in slightly stronger norms when the exact nature of the singularity of the solution is known. Our results lead to a boundary element method for two-dimensional screen problems in acoustics, which has twice the rate of convergence of the usual $h$ version with uniform mesh. An application to three-dimensional exterior problems is also analyzed.
\end{abstract}

1. Introduction. Over the last ten years there has been a spectacular increase in research and applications of boundary element techniques. There has been an explosion of books, as well as a series of international conferences [10], specially dedicated to boundary element methods (BEM). The state of the art of asymptotic error estimates of the $h$-version for BEM is described in several articles (see, for example, [24], [25]). There, the theoretical framework for both first-kind and second-kind integral equations is the theory of pseudodifferential operators. As observed in [21], for strongly elliptic pseudodifferential operators one has convergence of any Galerkin scheme with conforming boundary elements; also, there holds quasioptimality of the Galerkin error in the energy norm.

Almost all work on BEM has been performed with the $h$-version, where the degree $p$ of the elements is fixed, usually at a low value, typically $p=0,1,2$, and the accuracy is achieved by properly refining the mesh. Only recently has the $p$-version been introduced into the BEM [1], [2], [3], [26]. The $p$-version fixes the mesh and achieves the accuracy by increasing the degrees $p$ of the elements, uniformly or selectively. In the finite element method (FEM) the convergence of the $p$-version has been thoroughly investigated for one- and two-dimensional boundary value problems in a series of papers by Babuska and others [4], [5], [6], [7], [12]. Meanwhile, convergence results have also been derived for the $h-p$ version of the finite element method, which is a combination of the standard $h$-version and the p-version [4], [8], [13], [14].

In this paper, we prove the convergence of the $p$-version for some Galerkin boundary element schemes which use first-kind integral equations. In Section 2 we introduce the function spaces and corresponding norms used later on. In Subsection 3.1 we show that the rate of convergence of the $p$-version is an optimal one in the $H^{1 / 2}$ and $H^{-1 / 2}$-norms, generalizing known results for $H^{1}$ and $L^{2}$-norms. In Subsections $3.2,3.3$ we approximate singular functions by the $p$-version in the $\tilde{H}^{1 / 2}$

Received June 16, 1987; revised March 21, 1988.

1980 Mathematics Subject Classification (1985 Revision). Primary 65R20. 
and $\tilde{H}^{-1 / 2}$-norms, and we derive convergence rates which are twice the rate of the $h$-version with uniform mesh. In Section 4 we apply the approximation results of Section 3 to the Galerkin BEM for several integral equations which are strongly elliptic pseudodifferential equations. As examples, we consider the two-dimensional screen Neumann and Dirichlet problems in acoustics, where sharp regularity results for the solutions are available [22], [23]. Furthermore, we give first-kind boundary integral equations governing the exterior Dirichlet and Neumann problems of the three-dimensional Helmholtz equation, and we present the convergence rates for the $p$-version of the corresponding boundary element Galerkin schemes.

2. Notation. Let $\tilde{\Gamma}$ be a simply connected, bounded, smooth, closed curve in $\mathbf{R}^{2}$ and $\Gamma$ be a connected subset of $\tilde{\Gamma}$. By $C^{(k)}(\tilde{\Gamma}), 0 \leq k \leq \infty$ ( $k$ integer), we denote the space of all functions with continuous derivatives of order up to $k$ on $\tilde{\Gamma}$. As in [18], the Sobolev spaces $H^{s}(\tilde{\Gamma})$ are defined for $s \geq 0$ to be the restrictions of $H^{s+1 / 2}\left(\mathbf{R}^{2}\right)$ to $\tilde{\Gamma}$ and for $s<0$ by duality,

$$
H^{s}(\tilde{\Gamma})=\left(H^{-s}(\tilde{\Gamma})\right)^{\prime}
$$

with $H^{0}(\tilde{\Gamma})=L^{2}(\tilde{\Gamma})$. These spaces are used to define the corresponding spaces of distributions on $\Gamma$, namely, for any real $s$,

$$
\begin{gathered}
\tilde{H}^{s}(\Gamma)=\left\{u \in H^{s}(\tilde{\Gamma}): \operatorname{supp} u \subset \bar{\Gamma}\right\} \\
H^{s}(\Gamma)=\left\{\left.u\right|_{\Gamma}: u \in H^{s}(\tilde{\Gamma})\right\} .
\end{gathered}
$$

The above spaces are normed as follows. For $u$ defined on $\Gamma$, let $l u$ denote any extension of $u$ on $\tilde{\Gamma}$ and $u^{*}$ denote the zero extension of $u$ on $\tilde{\Gamma}$. Then

$$
\begin{gathered}
\|u\|_{\tilde{H}^{s}(\Gamma)}=\left\|u^{*}\right\|_{H^{s}(\tilde{\Gamma})} \\
\|u\|_{H^{s}(\Gamma)}=\inf \left\{\|l u\|_{H^{s}(\tilde{\Gamma})}: l u \in H^{s}(\tilde{\Gamma})\right\} .
\end{gathered}
$$

Note that for $s>1 / 2, s \neq$ integer $+1 / 2, \tilde{H}^{s}(\Gamma)$ is the usual $H_{0}^{s}(\Gamma)$ space, and for $-1 / 2<s<1 / 2, \tilde{H}^{s}(\Gamma)=H^{s}(\Gamma)$. For $s<-1 / 2, s \neq$ integer $+1 / 2$, we have $\tilde{H}^{s}(\Gamma)=\left(H^{-s}(\Gamma)\right)^{\prime}$. We will be particularly interested in the cases $s=1 / 2$ and $s=-1 / 2$. For $s=1 / 2$, the space $\tilde{H}^{1 / 2}(\Gamma)$ is also denoted by $H_{\infty}^{1 / 2}(\Gamma)$, with the equivalent norm (see [18])

$$
\|u\|_{H_{\infty}^{1 / 2}(\Gamma)}^{2}=\|u\|_{H^{1 / 2}(\Gamma)}^{2}+\left\|\left(1-x^{2}\right)^{-1 / 2} u\right\|_{H^{0}(\Gamma)}^{2},
$$

where for simplicity we have assumed $\Gamma=(-1,+1)$ (the general case can be treated by affine maps). and $x$ denotes the arc length. In terms of duality, the following relations hold

$$
H^{-1 / 2}(\Gamma)=\left(\tilde{H}^{1 / 2}(\Gamma)\right)^{\prime}, \quad \tilde{H}^{-1 / 2}(\Gamma)=\left(H^{1 / 2}(\Gamma)\right)^{\prime} .
$$

Let $\tilde{\Gamma}$ be of length $2 \pi$; then $H^{s}(\tilde{\Gamma})$ may be considered to be spaces of $2 \pi$-periodic functions. For $u \in H^{s}(\tilde{\Gamma})$, we may then write

$$
u(\xi)=\sum_{j=0}^{\infty} a_{j} \cos j \xi+\sum_{j=1}^{\infty} b_{j} \sin j \xi
$$


so that the $H^{s}(\tilde{\Gamma})$-norm may be equivalently defined by

$$
\|u\|_{H^{s}(\tilde{\Gamma})} \approx\left[\sum_{j=0}^{\infty} a_{j}^{2}\left(1+j^{2}\right)^{s}+\sum_{j=1}^{\infty} b_{j}^{2}\left(1+j^{2}\right)^{s}\right]^{1 / 2} .
$$

For $I$ a smooth open arc, we will define $\mathscr{P}_{p}(I)$ to be the set of all algebraic polynomials of degree less than or equal to $p$ in $x$, the arc length parameter. $\mathscr{P}_{p}^{0}(I)$ will denote the subset of polynomials vanishing at the end points of $I$.

Let us now subdivide $\tilde{\Gamma}$ into $N$ pieces, $\tilde{\Gamma}=\bigcup_{i=1}^{N} \bar{\Gamma}_{i}$, such that $\Gamma_{i}$ is a smooth open arc with end points $A_{i-1}, A_{i}\left(A_{0}=A_{N}\right)$. Then, for $p \geq 0, S_{p}(\tilde{\Gamma})$ will denote the set of all functions $u$ defined on $\tilde{\Gamma}$ such that the restriction $\left.u\right|_{\Gamma_{i}}$ to $\Gamma_{i}$ belongs to $\mathscr{P}_{p}\left(\Gamma_{i}\right)$. Moreover, we set for $p \geq 1$,

$$
V_{p}(\tilde{\Gamma})=S_{p}(\tilde{\Gamma}) \cap C^{(0)}(\tilde{\Gamma})
$$

We may assume that $\Gamma$ is partitioned analogously and define $S_{p}(\Gamma), V_{p}(\Gamma)$ as above. Then $S_{p}^{0}(\Gamma), V_{p}^{0}(\Gamma)$ will denote the subsets of functions that vanish at the end points of $\Gamma$.

Note that $S_{p}(\tilde{\Gamma})\left(S_{p}(\Gamma)\right)$ is a subset of $H^{-1 / 2}(\tilde{\Gamma})\left(H^{-1 / 2}(\Gamma)\right)$, while $V_{p}(\tilde{\Gamma})\left(V_{p}(\Gamma)\right)$ is a subset of $H^{1 / 2}(\tilde{\Gamma})\left(H^{1 / 2}(\Gamma)\right)$, and $V_{p}^{0}(\Gamma)$ is a subset of $\tilde{H}^{1 / 2}(\Gamma)$.

So far, we have dealt with the one-dimensional case. We will also be interested in a simply connected, bounded, smooth, closed surface $\tilde{\Gamma} \subset \mathbf{R}^{3}$. The definitions of $H^{s}(\tilde{\Gamma})$ are analogous to the previous case. We now assume that $\tilde{\Gamma}$ is partitioned into curvilinear quadrilaterals and triangles, i.e., $\tilde{\Gamma}=\bigcup_{i=1}^{n} \bar{\Gamma}_{i}$. Let $Q$ and $T$ be the reference square and triangle, respectively; then, $\Gamma_{i}=\mathscr{F}_{i}(Q)$ or $\mathscr{F}_{i}(T)$, where $\mathscr{F}_{i}$ is a smooth bijective mapping. We assume that the intersection of any two distinct $\Gamma_{i}$ 's is either the empty set or a common vertex or a common side.

By $\mathscr{P}_{p}^{1}(T)$ we will denote the set of all polynomials of total degree $\leq p$ on the triangle $T . \mathscr{P}_{p}^{2}(Q)$ will denote the set of all polynomials of degree $\leq p$ in each variable on $Q$. We define

$$
\begin{aligned}
S_{p}(\tilde{\Gamma})=\left\{u:\left.u\right|_{\Gamma_{i}}\left(\mathscr{F}_{i}(\xi)\right) \in \mathscr{P}_{p}^{1}(T) \text { if } \Gamma_{i}\right. \text { is a triangle and } \\
\left.\qquad\left.u\right|_{\Gamma_{i}}\left(\mathscr{F}_{i}(\xi)\right) \in \mathscr{P}_{p}^{2}(Q) \text { if } \Gamma_{i} \text { is a quadrilateral }\right\}
\end{aligned}
$$

and

$$
V_{p}(\tilde{\Gamma})=S_{p}(\tilde{\Gamma}) \cap C^{(0)}(\tilde{\Gamma})
$$

3. Approximation Theorems. In this section we will be interested in obtaining estimates for the approximation of functions in $H^{s}(\tilde{\Gamma}), \tilde{H}^{s}(\Gamma)$ and $H^{s}(\Gamma)$ by piecewise polynomials belonging to the polynomial subspaces introduced in the previous section.

3.1. Approximation of Functions in $H^{s}$. We first present some results for the case when $u$, the function being approximated, is known to lie in $H^{s}$. These will be used in the next section for approximating problems on closed curves and closed surfaces.

In what follows, $\tilde{\Gamma}$ will denote either a closed curve or a closed surface. 
THEOREM 3.1. Let $\gamma=\tilde{\Gamma}$ or $\Gamma$. Let $u \in H^{s}(\gamma), s>1 / 2$. Then for $p=1,2, \ldots$ there exists $u_{p} \in V_{p}(\gamma)$ such that

$$
\left\|u-u_{p}\right\|_{H^{1 / 2}(\gamma)} \leq C p^{-(s-1 / 2)}\|u\|_{H^{s}(\gamma)},
$$

where the constant $C$ is independent of $u$ and $p$, but depends on $s$ and the partition on $\gamma$. Moreover, for $u \in \tilde{H}^{s}(\Gamma)$,

$$
\left\|u-u_{p}\right\|_{\tilde{H}^{1 / 2}(\Gamma)} \leq C p^{-(s-1 / 2)} \log ^{1 / 2} p\|u\|_{\tilde{H}^{s}(\Gamma)} .
$$

Proof. The estimate (3.1) follows by interpolating the approximation estimates for the $p$-version obtained in the $H^{0}$ and $H^{1}$-norm (see [6]). In [7, Theorem 3.2], an alternative proof (for closed curves), using Chebyshev expansions, is provided. Moreover, (3.2) is also proved in this theorem, the procedure being similar to our proof of Theorem 3.3 in Subsection 3.2.

The above theorem provides estimates for the error of the best approximation in the $H^{1 / 2}$ and $\tilde{H}^{1 / 2}$-norms. The next theorem provides estimates in the $H^{-1 / 2}$. norm. It has been proved in [9] for $\gamma$ a closed curve, and it is included here for completeness.

THEOREM 3.2. Let $\gamma=\tilde{\Gamma}$ or $\Gamma, u \in H^{s}(\gamma), s \geq 0$. Then for $p=1,2,3, \ldots$ there exists $u_{p} \in V_{p}(\gamma)$ such that

$$
\left\|u-u_{p}\right\|_{\tilde{H}^{-1 / 2}(\gamma)} \leq C p^{-(s+1 / 2)}\|u\|_{H^{s}(\gamma)}
$$

where the constant $C$ is independent of $u$ and $p$, but depends upon $s$ and the grid on $\gamma$.

Proof. Let $u_{p} \in V_{p}(\gamma)$ satisfy

$$
\int_{\gamma} u_{p} \omega d \xi=\int_{\gamma} u \omega d \xi \text { for all } \omega \in V_{p}(\gamma) .
$$

Here, $u_{p}$ is the best $L_{2}$-approximation to $u$ in $V_{p}(\gamma)$, i.e.,

$$
\left\|u_{p}-u\right\|_{L_{2}(\gamma)}=\inf _{v \in V_{p}(\gamma)}\|v-u\|_{L_{2}(\gamma)} .
$$

Therefore, with $e=u-u_{p}$, we have (see [13])

$$
\|e\|_{H^{0}(\gamma)} \leq C p^{-s}\|u\|_{H^{s}(\gamma)} .
$$

Now, if $0 \neq v \in H^{1}(\gamma)$, then by (3.4),

$$
\begin{aligned}
\frac{\int_{\gamma} e v d \xi}{\|v\|_{H^{1}(\gamma)}} & =\frac{\int_{\gamma} e(v-y) d \xi}{\|v\|_{H^{1}(\gamma)}} \leq \frac{\|e\|_{H^{0}(\gamma)}\|v-y\|_{H^{0}(\gamma)}}{\|v\|_{H^{1}(\gamma)}} \\
& \leq C p^{-1}\|e\|_{H^{0}(\gamma)},
\end{aligned}
$$

where $y \in V_{p}(\gamma)$ satisfies

$$
\|v-y\|_{H^{0}(\gamma)} \leq C p^{-1}\|v\|_{H^{1}(\gamma)} .
$$

This yields

$$
\|e\|_{\left(H^{1}(\gamma)\right)^{\prime}} \leq C p^{-(s+1)}\|u\|_{H^{s}(\gamma)} .
$$


We obtain (3.3) by interpolating (3.5), (3.6) and using the fact that

$$
\begin{aligned}
\tilde{H}^{-1 / 2}(\gamma) & =\left(H^{1 / 2}(\gamma)\right)^{\prime}=\left(\left(H^{0}(\gamma), H^{1}(\gamma)\right)_{1 / 2}\right)^{\prime} \\
& =\left(H^{0}(\gamma),\left(H^{1}(\gamma)\right)^{\prime}\right)_{1 / 2},
\end{aligned}
$$

which follows from Theorem 6.2 in [18].

Remark 3.1. For $\gamma=\tilde{\Gamma}$, we have $\tilde{H}^{k}(\Gamma)=H^{k}(\Gamma)$. For $\gamma=\Gamma$, we have $\|\cdot\|_{H^{-1 / 2}(\Gamma)} \leq\|\cdot\|_{\tilde{H}^{-1 / 2}(\Gamma)}$. Hence, in either case, (3.3) yields

$$
\left\|u-u_{p}\right\|_{H^{-1 / 2}(\gamma)} \leq C p^{-(s+1 / 2)}\|u\|_{H^{s}(\gamma)} .
$$

Remark 3.2. Since $V_{p}(\gamma) \subset S_{p}(\gamma)$, we see that (3.3) and (3.7) also hold for some $u_{p} \in S_{p}(\gamma)$.

Remark 3.3. So far, we have assumed that $\tilde{\Gamma}$ and $\Gamma$ are smooth. The above theorems may also be modified to the case when $\tilde{\Gamma}$ and $\Gamma$ are only piecewise smooth.

3.2. $\tilde{H}^{1 / 2}$-Approximation of Singular Functions. We are interested here in approximating functions that are defined on the curve $\Gamma$ and have square root singularities at the end points. For simplicity, we consider a function $u$ defined on $I=[-1,+1]$ by

$$
u(x)=(x+1)^{1 / 2} \chi(x)
$$

where $\chi$ is a $C^{\infty}$ function satisfying

$$
\begin{aligned}
\chi(x) & =1, & & -1 \leq x \leq-1 / 2, \\
& =0, & & 1 / 2 \leq x \leq 1 .
\end{aligned}
$$

We consider the approximation of $u$ in the $\tilde{H}^{1 / 2}(I)$-norm by functions in $\mathscr{P}_{p}(I)$.

Let $\hat{I}=[-\pi, \pi]$. (We may consider $\hat{I}$ to be a closed circle.) Let $u$ be transformed to the periodic function $\hat{u}$ on $\hat{I}$ by the mapping $x=\cos \xi$, i.e., $\hat{u}(\xi)=u(x)$. Then we see that

$$
\hat{u}(\xi)=(1+\cos \xi)^{1 / 2} \chi(\cos \xi)=\sqrt{2} \chi(\cos \xi)(\cos (\xi / 2)) .
$$

The following lemma is taken from [7].

LEMMA 3.1. One has $\|u\|_{H^{1 / 2}(I)} \approx\|\hat{u}\|_{H^{1 / 2}(\hat{I})}$ for any $u \in H^{1 / 2}(I)$.

The main theorem of this section is the following.

THEOREM 3.3. Let $u$ be defined by (3.8). Then for $p=1,2, \ldots$ there exists a polynomial $u_{p}^{0}$ in $\mathscr{P}_{p}(I)$ such that

$$
u_{p}^{0}( \pm 1)=u( \pm 1)
$$

and

$$
\left\|u-u_{p}^{0}\right\|_{\tilde{H}^{1 / 2}(I)} \leq C p^{-1} \log ^{1 / 2} p .
$$

Proof. We first consider the image $\hat{u}$ of $u$, which (being even) may be written as

$$
\hat{u}(\xi)=\sum_{k=0}^{\infty} a_{k} \cos k \xi .
$$

Define

$$
u_{p}^{0}:=u_{p}+\bar{u}
$$


where $u_{p} \in \mathscr{P}_{p}(I)$ is defined in terms of the Chebyshev polynomials $T_{k}(x)=$ $\cos \left(k \cos ^{-1} x\right)$ of degrees less than or equal to $p$ by

$$
\hat{u}_{p}:=\sum_{k=0}^{p} a_{k} \cos k \xi=\sum_{k=0}^{p} a_{k} T_{k}(\cos \xi),
$$

and where $\bar{u}$ is a linear function such that $u_{p}^{0}$ satisfies the condition (3.10), i.e., such that

$$
\bar{u}( \pm 1)=\left(u-u_{p}\right)( \pm 1)=\left(\hat{u}-\hat{u}_{p}\right)\left(\cos ^{-1}( \pm 1)\right) .
$$

We now estimate the coefficients $a_{k}$ in (3.12). We have

$$
\begin{aligned}
a_{k} & =C \int_{0}^{\pi} \hat{u} \cos k \xi d \xi=C \int_{0}^{\pi} \chi(\cos \xi) \cos \left(\frac{\xi}{2}\right) \cos k \xi d \xi \\
& =C \int_{0}^{\pi} \chi(\cos \xi)\left[\cos \left(\left(\frac{2 k+1}{2}\right) \xi\right)+\cos \left(\left(\frac{2 k-1}{2}\right) \xi\right)\right] d \xi
\end{aligned}
$$

Here, $C$ may represent different constants. Integrating by parts gives

$$
\begin{aligned}
a_{k}=C\left\{\left[\chi(\cos \xi)\left(\sin \left(\left(\frac{2 k+1}{2}\right) \xi\right) \cdot \frac{1}{2 k+1}+\sin \left(\left(\frac{2 k-1}{2}\right) \xi\right) \cdot \frac{1}{2 k-1}\right)\right]_{0}^{\pi}\right. \\
+\int_{0}^{\pi} \chi^{\prime}(\cos \xi) \sin \xi\left[\sin \left(\left(\frac{2 k+1}{2}\right) \xi\right) \cdot \frac{1}{2 k+1}\right. \\
\left.\left.\quad+\sin \left(\left(\frac{2 k-1}{2}\right) \xi\right) \frac{1}{2 k-1}\right] d \xi\right\} .
\end{aligned}
$$

Using $\chi^{\prime}( \pm 1)=0$, further integration by parts yields

$$
\begin{aligned}
\left|a_{k}\right| \leq C\left\{\frac{1}{k^{2}}+\mid \int_{0}^{\pi} \chi^{\prime}(\cos \xi)[\right. & \cos \left(\left(k-\frac{1}{2}\right) \xi\right) \frac{1}{2 k+1} \\
& -\cos \left(\left(k+\frac{3}{2}\right) \xi\right) \frac{1}{2 k+1} \\
& +\cos \left(\left(k-\frac{3}{2}\right) \xi\right) \frac{1}{2 k-1} \\
\leq C\left\{\frac{1}{k^{2}}+\mid \int_{0}^{\pi} \chi^{\prime \prime}(\cos \xi) \sin \xi\right. & {\left[\frac{\sin \left(k-\frac{1}{2}\right) \xi}{(2 k+1)(2 k-1)}-\frac{\sin \left(k+\frac{3}{2}\right) \xi}{(2 k+1)(2 k+3)}\right.} \\
& \left.\left.\quad+\frac{\sin \left(k-\frac{3}{2}\right) \xi}{(2 k-1)(2 k-3)}-\frac{\sin \left(k+\frac{1}{2}\right) \xi}{(2 k-1)(2 k+1)}\right] d \xi \mid\right\} .
\end{aligned}
$$

Now, since $\chi^{\prime \prime}$ and all the sine functions are bounded independent of $k$, we obtain

$$
\left|a_{k}\right| \leq \frac{C}{k^{2}} \text {. }
$$

We now estimate $\left\|u-u_{p}\right\|_{H^{1 / 2}(I)}$. By Lemma 3.1, (2.5), (3.12), and (3.14), we have

$$
\begin{aligned}
\left\|u-u_{p}\right\|_{H^{1 / 2}(I)}^{2} & =\left\|\hat{u}-\hat{u}_{p}\right\|_{H^{1 / 2}(\hat{I})}^{2}=C \sum_{k=p+1}^{\infty} a_{k}^{2}\left(1+k^{2}\right)^{1 / 2} \\
& \leq C \sum_{p+1}^{\infty} \frac{\left(1+k^{2}\right)^{1 / 2}}{k^{4}}
\end{aligned}
$$


which behaves like

$$
\int_{p+1}^{\infty} \frac{C}{x^{3}} d x=\frac{C}{p^{2}}
$$

Hence,

$$
\left\|u-u_{p}\right\|_{H^{1 / 2}(I)} \leq \frac{C}{p} .
$$

Next, we estimate $\|\bar{u}\|_{H^{1 / 2}(I)}$. Since $\bar{u}$ is linear,

$$
\|\bar{u}\|_{H^{1 / 2}(I)} \leq C\{|u(+1)|+|u(-1)|\} .
$$

Now for any $x$, by (3.12), (3.14),

$$
\left|\left(u-u_{p}\right)(x)\right| \leq \sum_{k=p+1}^{\infty}\left|a_{k}\right| \leq \sum_{k=p+1}^{\infty} \frac{C}{k^{2}} \leq \frac{C}{p} .
$$

Using (3.15), (3.19), and (3.18), we see that

$$
\|\bar{u}\|_{H^{1 / 2}(I)} \leq \frac{C}{p}
$$

which combined with (3.17) yields

$$
\left\|u-u_{p}^{0}\right\|_{H^{1 / 2}(I)} \leq \frac{C}{p} .
$$

By (2.3), we know that

$$
\left\|u-u_{p}^{0}\right\|_{\tilde{H}^{1 / 2}(I)} \leq\left\|u-u_{p}^{0}\right\|_{H^{1 / 2}(I)}+\left\|\left(1-x^{2}\right)^{-1 / 2}\left(u-u_{p}^{0}\right)\right\|_{H^{0}(I)} .
$$

Hence we must bound the second term. We have

$$
\begin{aligned}
& \int_{-1}^{+1}\left(1-x^{2}\right)^{-1}\left(u-u_{p}^{0}\right)^{2} d x \\
& \quad=\left(\int_{0}^{1 / p}+\int_{1 / p}^{\pi-1 / p}+\int_{\pi-1 / p}^{\pi}\right)\left(\hat{u}-\hat{u}_{p}^{0}\right)^{2}(\sin \xi)^{-1} d \xi .
\end{aligned}
$$

Now $1 / \sin \xi$ is bounded on $[1 / p, \pi-1 / p]$. Hence, using (3.19),

$$
\begin{aligned}
\int_{1 / p}^{\pi-1 / p}\left(\hat{u}-\hat{u}_{p}^{0}\right)^{2}(\sin \xi)^{-1} d \xi & \leq \frac{C}{p^{2}} \int_{1 / p}^{\pi-1 / p}(\sin \xi)^{-1} d \xi \\
& \leq \frac{C}{p^{2}} \log p .
\end{aligned}
$$

Also, let $[a]$ denote the integral part of $a$. Then it may be verified that with

$$
\hat{\bar{u}}:=\sum_{j=[(p+2) / 2]}^{\infty} a_{2 j}+\sum_{j=[(p+1) / 2]}^{\infty} a_{2 j+1} \cos \xi
$$

we obtain with (3.13)

$\hat{u}(\xi)-\hat{u}_{p}^{0}(\xi)=\sum_{j=[(p+2) / 2]}^{\infty} a_{2 j}(\cos 2 j \xi-1)+\sum_{j=[(p+1) / 2]}^{\infty} a_{2 j+1}(\cos (2 j+1) \xi-\cos \xi)$,

which satisfies

$$
\left(\hat{u}-\hat{u}_{p}^{0}\right)\left(\cos ^{-1}( \pm 1)\right)=0
$$


as required in (3.10). Hence,

$$
\begin{aligned}
\left(\hat{u}-\hat{u}_{p}^{0}\right)^{2} \leq C & \left\{\left(\sum_{j=[(p+2) / 2]}^{\infty} a_{2 j}(\cos 2 j \xi-1)\right)^{2}\right. \\
& \left.+\left(\sum_{j=[(p+1) / 2]}^{\infty} a_{2 j+1}(\cos (2 j+1) \xi-\cos \xi)\right)^{2}\right\} \\
\leq C & \left\{\left(\sum_{j=[(p+2) / 2]}^{\infty} a_{2 j} \sin ^{2} j \xi\right)^{2}\right. \\
& \left.+\left(\sum_{j=[(p+1) / 2]}^{\infty} a_{2 j+1} \sin (j+1) \xi \sin j \xi\right)^{2}\right\}
\end{aligned}
$$

so that

$$
\begin{aligned}
& \int_{0}^{1 / p}\left(\hat{u}-\hat{u}_{p}^{0}\right)^{2}(\sin \xi)^{-1} d \xi \\
& \leq C\left\{\int_{0}^{1 / p}\left(\sum_{j=[(p+2) / 2]}^{\infty} a_{2 j} \sin ^{2} j \xi\right)^{2}(\sin \xi)^{-1} d \xi\right. \\
&\left.+\int_{0}^{1 / p}\left(\sum_{j=[(p+1) / 2]}^{\infty} a_{2 j+1} \sin (j+1) \xi \sin j \xi\right)^{2}(\sin \xi)^{-1} d \xi\right\} .
\end{aligned}
$$

Now, $\sin j \xi \leq j \xi$, so that for any $\varepsilon>0$,

$$
\sin ^{2} j \xi \leq|\sin j \xi|^{\varepsilon}|\sin j \xi|^{2-\varepsilon} \leq(j \xi)^{\varepsilon} \quad \text { for } 0<\xi<1 / p .
$$

Hence, using (3.16), the first term on the right side of (3.25) is bounded by

$$
\begin{aligned}
\int_{0}^{1 / p}\left(\sum_{j=[(p+2) / 2]}^{\infty} \frac{j^{\varepsilon}}{j^{2}}\right)^{2} \cdot \frac{\xi^{2 \varepsilon}}{\sin \xi} d \xi & \leq \frac{C}{p^{2(1-\varepsilon)}} \int_{0}^{1 / p} \xi^{2 \varepsilon-1} d \xi \\
& \leq \frac{C}{p^{2(1-\varepsilon)}} \cdot p^{-2 \varepsilon}=\frac{C}{p^{2}}
\end{aligned}
$$

The second term may be similarly bounded, as may the term

$$
\int_{\pi-1 / p}^{\pi}\left(\hat{u}-\hat{u}_{p}^{0}\right)^{2}(\sin \xi)^{-1} d \xi
$$

Using this with (3.23), (3.24) gives

$$
\left\|\left(1-x^{2}\right)^{-1 / 2}\left(u-u_{p}^{0}\right)\right\|_{H^{0}(I)} \leq \frac{C \log ^{1 / 2} p}{p},
$$

which combined with (3.21)-(3.22) yields (3.11).

In Section 4 we will use Theorems 3.1 and 3.3 to bound the error made when a function that is smooth in the interior of $\Gamma$ and behaves like (3.8) at the end points is approximated by functions in $V_{p}(\Gamma)$. 
3.3. $\tilde{H}^{-1 / 2}$-Approximation of Singular Functions. In this section we consider the approximation of functions $u$ defined on $I=[-1,+1]$ of the form

$$
u(x)=(x+1)^{-1 / 2} \chi(x),
$$

where $\chi$ is as before. We are now interested in approximating $u$ in the $\tilde{H}^{-1 / 2}(I)$ norm by functions in $\mathscr{P}_{p}(I)$. To this end, we first prove the following lemma.

LEMMA 3.2. Let $f \in \tilde{H}^{1 / 2}(I)$. Then $f^{\prime} \in \tilde{H}^{-1 / 2}(I)$ and

$$
\left\|f^{\prime}\right\|_{\tilde{H}^{-1 / 2}(I)} \leq C\|f\|_{\tilde{H}^{1 / 2}(I)} .
$$

Proof. Let $\psi \in C_{0}^{\infty}(-1,1)$. Define $\psi^{*}$ to be the extension by 0 of $\psi$ to $\mathbf{R}$. Then it may be easily seen that $\psi^{* \prime}=\psi^{\prime *}$, so that

$$
\begin{aligned}
\left\|\psi^{\prime}\right\|_{H^{-1 / 2}(I)} & =\left\|\psi^{\prime *}\right\|_{H^{-1 / 2}(\mathbf{R})}=\left\|\psi^{* \prime}\right\|_{H^{-1 / 2}(\mathbf{R})} \\
& \leq C\left\|\psi^{*}\right\|_{H^{1 / 2}(\mathbf{R})} \leq C\|\psi\|_{H^{1 / 2}(I)}
\end{aligned}
$$

since $\psi \in C_{0}^{\infty}(-1,1)$. (The inequality $(3.28)$ can be verified taking Fourier transforms, for instance.)

Now let $f \in \tilde{H}^{1 / 2}(I)$. We use the following definition, from [11], for the $\tilde{H}^{-1 / 2}(I)$-norm:

Hence, with

$$
\left\|f^{\prime}\right\|_{\tilde{H}^{-1 / 2}(I)}=\sup _{\psi \in C_{0}^{\infty}(-1,1)} \frac{\left|\left\langle f^{\prime}, \psi\right\rangle_{L^{2}(I)}\right|}{\|\psi\|_{H^{1 / 2}(I)}} .
$$

$$
\left\langle f^{\prime}, \psi\right\rangle_{L^{2}(I)}=-\left\langle f, \psi^{\prime}\right\rangle_{L^{2}(I)}
$$

we obtain

$$
\begin{aligned}
\left\|f^{\prime}\right\|_{\tilde{H}^{-1 / 2}(I)} & =\sup _{\psi \in C_{0}^{\infty}(-1,1)} \frac{\left|\left\langle f, \psi^{\prime}\right\rangle_{L^{2}(I)}\right|}{\|\psi\|_{H^{1 / 2}(I)}} \\
& \leq \sup _{\psi \in C_{0}^{\infty}(-1,1)} \frac{\|f\|_{\tilde{H}^{1 / 2}(I)}\left\|\psi^{\prime}\right\|_{H^{-1 / 2}(I)}}{\|\psi\|_{H^{1 / 2}(I)}} \\
& \leq C\|f\|_{\tilde{H}^{1 / 2}(I)}
\end{aligned}
$$

by (3.28). This proves the lemma.

With Lemma 3.2 we obtain the following analog to Theorem 3.3.

THEOREM 3.4. Let $u$ be defined by (3.26). Then there exists a polynomial $u_{p}$ in $\mathscr{P}_{p}(I)$ such that

$$
\left\|u-u_{p}\right\|_{\tilde{H}^{-1 / 2}(I)} \leq C p^{-1} \log ^{1 / 2} p
$$

Proof. Let

$$
w=\int_{-1}^{x} u(t) d t=2(x+1)^{1 / 2} \chi(x)-2 \int_{-1}^{x}(t+1)^{1 / 2} \chi^{\prime}(t) d t=w_{1}+w_{2}
$$

By Theorem 3.3, there exists $v_{p}^{1} \in \mathscr{P}_{p}(I)$ satisfying

$$
\left\|w_{1}-v_{p}^{1}\right\|_{\tilde{H}^{1 / 2}(I)} \leq C p^{-1} \log ^{1 / 2} p .
$$

Also, since $\chi(x)$ is smooth, $w_{2}$ lies in $H^{2-\varepsilon}$ for any $\varepsilon>0$. Applying Theorem 3.1, there exists $v_{p}^{2} \in \mathscr{P}_{p}(I)$ satisfying

$$
\begin{aligned}
\left\|w_{2}-v_{p}^{2}\right\|_{\tilde{H}^{1 / 2}(I)} & \leq C p^{-(2-\varepsilon-1 / 2)} \log ^{1 / 2} p\left\|w_{2}\right\|_{H^{2-\varepsilon}(I)} \\
& \leq C p^{-1} \log ^{1 / 2} p .
\end{aligned}
$$


Taking $v_{p}=v_{p}^{1}+v_{p}^{2}$, we have

$$
\left\|w-v_{p}\right\|_{\tilde{H}^{1 / 2}(I)} \leq C p^{-1} \log ^{1 / 2} p .
$$

Finally, using Lemma 3.2 and taking $u_{p}=v_{p}^{\prime}$, we obtain (3.29).

4. The $p$-Version for Boundary Elements. Before we apply the approximation results of Section 3 to the Galerkin solutions of some integral equations of the first kind, let us recall some basic facts on the Galerkin method. The key to the error analysis of Galerkin's method is the following result by Hildebrandt and Wienholtz [15] (see also [11], [21]).

LEMMA 4.1. Let $H$ be a Hilbert space with dual $H^{\prime}$ (not necessarily identified with $H$ ), and let $A$ be injective and continuous from $H$ into $H^{\prime}$ satisfying a Garding inequality. Let $u \in H$ denote the solution of

$$
A u=f,
$$

where $f \in H^{\prime}$, and let $u_{N} \in S_{N} \subset H$ denote the solution of the Galerkin equations

$$
\left\langle A u_{N}, v\right\rangle=\langle f, v\rangle \text { for all } v \in S_{N} \subset H \text {. }
$$

Furthermore, assume that for any $\phi \in H$ there exists $\phi_{N} \in S_{N}$ with $\phi=\lim _{N \rightarrow \infty} \phi_{N}$ in $H$. Then, for $N$ large enough, the Galerkin equations (4.2) are uniquely solvable and with a constant $C$ independent of $u, u_{N}$ and $N$, there holds the error estimate

$$
\left\|u-u_{N}\right\| \leq C \inf \left\{\left\|u-v_{N}\right\|: v_{N} \in S_{N}\right\},
$$

where $\|\cdot\|$ denotes the norm in $H$.

Next, we list several boundary value problems which can be reduced to strongly elliptic integral equations, i.e., the corresponding integral operators satisfy a Gårding inequality in appropriate Sobolev spaces. Therefore, by Lemma 4.1, the corresponding boundary element Galerkin methods converge, and the quasioptimality (4.3) holds, which together with the approximation results of Section 3 leads to error estimates for the $p$-version.

The Neumann screen problem in acoustics describes the scattering of a plane wave at a hard obstacle $\Gamma$. Here, $\Gamma$ is given by an oriented open arc, being a finite piece of a smooth curve in $\mathbf{R}^{2}$. The orientation defines the normal vector $n$ pointing to the side $\Gamma_{2}$ (see Figure 1). The opposite side of $\Gamma$ will be denoted by $\Gamma_{1}$. The scattering problem gives rise to the problem: Find the pressure amplitude field $u \in H^{1}\left(\Omega_{\Gamma} \cap K\right)$ for every compact subset $K \subset \mathbf{R}^{2}$ satisfying

$$
\begin{array}{cc}
\left(\Delta+k^{2}\right) u=0 & \text { in } \Omega_{\Gamma}=\mathbf{R}^{2} \backslash \bar{\Gamma} \\
\left.\frac{\partial u}{\partial n}\right|_{\Gamma_{1}}=g_{1}, & \left.\frac{\partial u}{\partial n}\right|_{\Gamma_{2}}=g_{2} .
\end{array}
$$

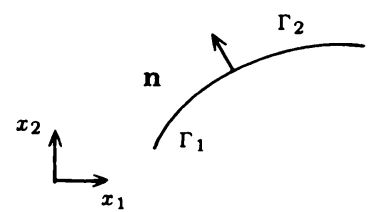

FIGURE 1

Here, $k \neq 0, \operatorname{Im} k \geq 0$, and $g_{1}, g_{2} \in H^{-1 / 2}(\Gamma)$ are given with $g:=g_{1}-g_{2} \in$ $\tilde{H}^{-1 / 2}(\Gamma)$. In addition, we require the Sommerfeld radiation condition

$$
\frac{\partial u}{\partial r}-i k u=o\left(r^{-1 / 2}\right) \text { and } u=O\left(r^{-1 / 2}\right) \quad \text { as } r=|x| \rightarrow \infty .
$$

From [23] we know that for $\operatorname{Im} k \geq 0, k \neq 0$, the problem (4.4), (4.5) has no eigensolutions, and furthermore it can be reduced to a hypersingular integral equation on $\Gamma$. 
THEOREM 4.1 [23]. Let $g_{1}, g_{2}$ and $k$ be given as above. Then there holds: (i) $u \in H^{1}\left(\Omega_{\Gamma} \cap K\right)$ for every compact subset $K \subset \mathbf{R}^{2}$ solves (4.4), (4.5) if and only if the jump $\left.[u]\right|_{\Gamma} \in \tilde{H}^{1 / 2}(\Gamma)$ satisfies the integral equation

$$
D[u](z):=-2 \int_{\Gamma}[u](\varsigma) \frac{\partial^{2}}{\partial n_{z} \partial n_{\varsigma}} \Phi(z, \varsigma) d s_{\varsigma}=f(z), \quad z \in \Gamma,
$$

with

$$
f(z):=g_{1}(z)+g_{2}(z)+2 \int_{\Gamma}\left(g_{1}(\varsigma)-g_{2}(\varsigma)\right) \frac{\partial}{\partial n_{z}} \Phi(z, \varsigma) d s_{\zeta}
$$

where

$$
\Phi(z, \varsigma):=\frac{-i}{4} H_{0}^{(1)}(k|z-\varsigma|)
$$

and $H_{0}^{(1)}$ is the Hankel function of the first kind and order zero. (ii) There exists exactly one solution $\psi \in \tilde{H}^{1 / 2}(\Gamma), \psi=\left.[u]\right|_{\Gamma}$ of (4.6).

The proof of the assertion (ii) in [23] hinges on the fact that $D$ is a strongly elliptic pseudodifferential operator of order 1 . Therefore, there exists a constant $\gamma_{1}>0$ and a compact mapping $C_{1}: \tilde{H}^{1 / 2}(\Gamma) \rightarrow H^{-1 / 2}(\Gamma)$ such that

$$
\operatorname{Re}\left\langle\left(D+C_{1}\right) \psi, \psi\right\rangle_{L^{2}(\Gamma)} \geq \gamma_{1}\|\psi\|_{\tilde{H}^{1 / 2}(\Gamma)}^{2}
$$

for every $\psi \in \tilde{H}^{1 / 2}(\Gamma)$. This yields that $D$ is a Fredholm operator of index zero, and the bijectivity of $D$ thus follows from its injectivity, which is guaranteed by the above assumptions on the wave number $k$. Note that the assumptions on $g_{1}, g_{2}$ imply $f \in H^{-1 / 2}(\Gamma)$.

Using localization and Mellin transformation, Stephan and Wendland derive in [23] the following explicit regularity result for the solution of (4.6) near the end points $z_{1}, z_{2}$ of $\Gamma$.

LEMMA 4.2 [23]. For $0 \leq \sigma<1 / 2$ let $g_{j} \in H^{1 / 2+\sigma}(\Gamma), j=1,2$, be given. Then the solution $\left.[u]\right|_{\Gamma} \in \tilde{H}^{1 / 2}(\Gamma)$ of the integral equation (4.6) has the form

$$
\left.[u]\right|_{\Gamma}=\sum_{i=1}^{2} \alpha_{i} \rho_{i}^{1 / 2} \chi_{i}+v_{0} \quad \text { with } v_{0} \in \tilde{H}^{3 / 2+\sigma}(\Gamma), \alpha_{i} \in \mathbf{R} .
$$

Here, $\rho_{i}$ denotes the Euclidean distance between $z \in \Gamma$ and the end point $z_{i}$ of $\Gamma$. $\chi_{i}$ is a $C^{\infty}$ cutoff function with $0 \leq \chi_{i} \leq 1$ and $\chi_{i}=1$ near to $z_{i}, \chi_{i}=0$ at the opposite end point, $i=1,2$.

The p-version Galerkin method for the hypersingular integral equation (4.6) reads: Find $v_{p} \in V_{p}^{0}(\Gamma)$ such that, with $f \in H^{-1 / 2}(\Gamma)$ given by (4.7), for all $\phi_{p} \in V_{p}^{0}(\Gamma)$ there holds

$$
\left\langle D v_{p}, \phi_{p}\right\rangle_{L^{2}(\Gamma)}=\left\langle f, \phi_{p}\right\rangle_{L^{2}(\Gamma)}
$$

Here, $V_{p}^{0}(\Gamma)$ denotes the set of continuous, piecewise polynomials of degree $\leq p$ which vanish at the end points of $\Gamma$, as introduced in Section 2. Note $V_{p}^{0}(\Gamma) \subset$ $\tilde{H}^{1 / 2}(\Gamma)$. There holds the following convergence result for the Galerkin scheme (4.11). 
THEOREM 4.2. Let $p$ be sufficiently large. Then the Galerkin equations (4.11) are uniquely solvable and for the error between the exact solution $\left.[u]\right|_{\Gamma} \in \tilde{H}^{1 / 2}(\Gamma)$ of (4.6) and the Galerkin solution $v_{p} \in V_{p}^{0}(\Gamma)$ we have

$$
\left\|[u]-v_{p}\right\|_{\tilde{H}^{1 / 2}(\Gamma)} \leq C p^{-1} \log ^{1 / 2} p,
$$

where the constant $C$ depends on $u$ but is independent of $p$.

Proof. We observe that the operator $D$ in (4.6) fulfills the requirements on $A$ in Lemma 4.1 with $H:=\tilde{H}^{1 / 2}(\Gamma)$, and $H^{\prime}=H^{-1 / 2}(\Gamma)$ since $D$ satisfies the Garding inequality (4.9) and $D$ is bijective from $\tilde{H}^{1 / 2}(\Gamma)$ onto $H^{-1 / 2}(\Gamma)$ by virtue of Theorem 4.1(ii). On the other hand, $\left\{V_{p}^{0}(\Gamma)\right\}$ is a sequence of approximating subspaces of $\tilde{H}^{1 / 2}(\Gamma)$ as $p \rightarrow \infty$, and therefore $V_{p}^{0}(\Gamma)$ is a candidate for the subspace $S_{N}$ in Lemma 4.1 with $p$ instead of $N$. Thus, the convergence of the $p$-version for the Galerkin procedure (4.11) is an immediate consequence of Lemma 4.1. The rate of convergence in (4.12) follows from the quasioptimality (4.3) together with the regularity result (4.10), where the approximation result (3.11) is used to approximate the singular part in (4.10), and Theorem 3.1 is used to approximate the regular part $v_{0}$.

Remark 4.1. (i) The decomposition (4.10) shows that the exact solution $\psi=\left[\left.u\right|_{\Gamma}\right.$ of the integral equation (4.6) belongs to $H^{1-\varepsilon}(\Gamma)$ for any $\varepsilon>0$. Therefore, the $h$-version of the Galerkin procedure for Eq. (4.6) gives only an estimate of order $O\left(h^{1 / 2}\right)$ for the Galerkin error, if a uniform mesh is used.

(ii) Application of the estimate (3.2) to the quasioptimality estimate (4.3) gives, with $\psi \in \tilde{H}^{1-\varepsilon}(\Gamma)$,

$$
\left\|\psi-v_{p}\right\|_{\tilde{H}^{1 / 2}(\Gamma)} \leq C p^{-1 / 2+\varepsilon} \log ^{1 / 2} p\|\psi\|_{\tilde{H}^{1-\varepsilon}(\Gamma)} .
$$

The better estimate (4.12) follows from Theorem 3.3.

The Dirichlet screen problem in acoustics describes the scattering of a plane wave at a soft obstacle $\Gamma$. With $\Gamma$ being an open arc as introduced above, the scattering problem becomes: Find the pressure amplitude field $u \in H^{1}\left(\Omega_{\Gamma} \cap K\right)$ for every compact subset $K \subset \mathbf{R}^{2}$ satisfying

$$
\left(\Delta+k^{2}\right) u=0 \quad \text { in } \Omega_{\Gamma}=\mathbf{R}^{2} \backslash \bar{\Gamma}, \quad u=g \quad \text { on } \Gamma,
$$

together with the radiation condition (4.5) for given $g \in H^{1 / 2}(\Gamma)$ and $k \neq 0$, $\operatorname{Im} k \geq 0$.

We know from [22] that with the above restrictions on the wave number $k$ the Dirichlet problem (4.13), (4.5) has no eigensolutions. Furthermore, this Dirichlet problem can be reduced to a weakly singular integral equation on $\Gamma[22]$.

THEOREM $4.3[22]$. Let $g \in H^{1 / 2}(\Gamma)$ be given and $k \neq 0, \operatorname{Im} k \geq 0$. Then there holds: (i) $u \in H^{1}\left(\Omega_{\Gamma} \cap K\right)$ for every compact subset $K \subset \mathbf{R}^{2}$ solves (4.13), (4.5) if and only if the jump $\left.[\partial u / \partial n]\right|_{\Gamma} \in \tilde{H}^{-1 / 2}(\Gamma)$ satisfies the integral equation

$$
V\left[\frac{\partial u}{\partial n}\right](z):=-2 \int_{\Gamma}\left[\frac{\partial u}{\partial n}\right](\varsigma) \Phi(z, \varsigma) d s_{\varsigma}=2 g(z), \quad z \in \Gamma,
$$

where $\Phi$ is given in (4.8).

(ii) There exists exactly one solution $\psi \in \tilde{H}^{-1 / 2}(\Gamma), \psi=\left.[\partial n / \partial n]\right|_{\Gamma}$ of (4.14). 
(iii) Let $g \in H^{3 / 2+\sigma}(\Gamma), 0 \leq \sigma<1 / 2$, be given. Then with the notation of Lemma 4.2 the solution $\left.[\partial u / \partial n]\right|_{\Gamma} \in \tilde{H}^{-1 / 2}(\Gamma)$ of (4.14) has the form

$$
\left.\left[\frac{\partial u}{\partial n}\right]\right|_{\Gamma}=\sum_{i=1}^{2} \alpha_{i} \rho_{i}^{-1 / 2} \chi_{i}+\psi_{0} \quad \text { with } \psi_{0} \in \tilde{H}^{1 / 2+\sigma}(\Gamma), \alpha_{i} \in \mathbf{R}
$$

The proof of assertion (ii) in [22] uses the fact that the single-layer potential operator $V$ is a strongly elliptic pseudodifferential operator of order -1 . Therefore, there holds with a constant $\gamma_{2}$ and a compact operator $C_{2}: \tilde{H}^{-1 / 2}(\Gamma) \rightarrow H^{1 / 2}(\Gamma)$ the Gårding inequality

$$
\operatorname{Re}\left\langle\left(V+C_{2}\right) \psi, \psi\right\rangle_{L^{2}(\Gamma)} \geq \gamma_{2}\|\psi\|_{\tilde{H}^{-1 / 2}(\Gamma)}^{2}
$$

for any $\psi \in \tilde{H}^{-1 / 2}(\Gamma)$.

The decomposition (4.15) is obtained in [22] by localizing the weakly singular integral equation (4.14) and applying the Mellin transformation. The explicit form (4.15) of the solution near the end points $z_{i}, i=1,2$, allows us to derive optimal error estimates for the Galerkin solution.

The p-version Galerkin method for the weakly singular integral equation (4.14) reads: Find $\psi_{p} \in S_{p}(\Gamma)$ such that with $g \in H^{1 / 2}(\Gamma)$

$$
\left\langle V \psi_{p}, \phi_{p}\right\rangle=\left\langle 2 g, \phi_{p}\right\rangle \text { for all } \phi_{p} \in S_{p}(\Gamma) \text {. }
$$

Here, $S_{p}(\Gamma)$ denotes the set of piecewise polynomials of degree $\leq p$ subordinate to a partitioning of $\Gamma$ as introduced in Section 2. Note $S_{p}(\Gamma) \subset \tilde{H}^{-1 / 2}(\Gamma)$.

THEOREM 4.4. Let $p$ be sufficiently large. Then the Galerkin equations (4.17) are uniquely solvable, and the error between the exact solution $\psi$ of (4.14) and the Galerkin solution $\psi_{p} \in S_{p}(\Gamma)$ of (4.17) satisfies

$$
\left\|\psi-\psi_{p}\right\|_{\tilde{H}^{-1 / 2}(\Gamma)} \leq C p^{-1} \log ^{1 / 2} p
$$

with a constant $C$ independent of $p$.

Proof. Owing to the Garding inequality (4.16), application of Lemma 4.1 yields for the choices $A=V$ and $H=\tilde{H}^{-1 / 2}(\Gamma)$ with $H^{\prime}=H^{1 / 2}(\Gamma)$ the convergence of the Galerkin scheme (4.17). Note that $\left\{S_{p}(\Gamma)\right\}$ as introduced in Section 2 is a sequence of approximating subspaces for $\tilde{H}^{-1 / 2}(\Gamma)$ as $p \rightarrow \infty$. The estimate (4.18) follows from the quasioptimality (4.3) together with the regularity result (4.15), where (3.29) is used to approximate the singular part in (4.15), and Theorem 3.2 is used to approximate the regular part $\psi_{0}$.

The exterior Neumann (Dirichlet) problem in acoustics describes the scattering of a plane wave at a hard (soft) obstacle given by a bounded domain $\Omega$ in $\mathbf{R}^{3}$. For simplicity we assume that the boundary $\tilde{\Gamma}$ of $\Omega$ is a closed, smooth, simply connected surface. Then the scattering problem leads to the problem: Find $u \in H_{\text {loc }}^{1}\left(\mathbf{R}^{3} \backslash \bar{\Omega}\right)$ satisfying

$$
\begin{aligned}
& \left(\Delta+k^{2}\right) u=0 \quad \text { in } \mathbf{R}^{3} \backslash \bar{\Omega} \\
& \frac{\partial u}{\partial n}=g \quad \text { on } \tilde{\Gamma}(\text { Neumann })
\end{aligned}
$$

or

$$
u=f \quad \text { on } \tilde{\Gamma} \text { (Dirichlet) }
$$


for $k \neq 0, \operatorname{Im} k \geq 0$, together with the radiation condition

$$
\frac{\partial u}{\partial r}-i k u=o\left(r^{-1}\right), \quad u=O\left(r^{-1}\right) \quad \text { as } r=|x| \rightarrow \infty .
$$

Here we make the general assumption:

In the exterior Neumann (Dirichlet) problem in $\mathbf{R}^{3} \backslash \bar{\Omega}$ let $k^{2}$ be different from the eigenvalues of the interior Dirichlet (Neumann) problem in $\Omega$.

The restriction to the three-dimensional case is only for simplicity. Of course, we can derive analogous results also for the corresponding 2-D problems. Easy modifications of the procedure in [16], [20] lead directly to a boundary integral equation method for the Neumann and the Dirichlet problem. One immediately obtains existence and uniqueness results analogous to Theorems 4.1 and 4.3. Let us first consider again the Neumann problem (4.19), (4.20), (4.22).

THEOREM 4.5. Let $g \in H^{-1 / 2}(\tilde{\Gamma})$ be given with $\int_{\tilde{\Gamma}} g d s=0$. Then, with $k$ as above, there holds:

(i) $u \in H_{\text {loc }}^{1}\left(\mathbf{R}^{3} \backslash \bar{\Omega}\right)$ solves (4.19), (4.20), (4.22) if and only if $u \in H^{1 / 2}(\tilde{\Gamma})$ satisfies the integral equation

$$
D u(z):=-2 \int_{\tilde{\Gamma}} u(\varsigma) \frac{\partial^{2}}{\partial n_{z} \partial n_{\zeta}} \Phi(z, \varsigma) d s_{z}=\tilde{g}(z), \quad z \in \Gamma
$$

where

$$
\Phi(z, \varsigma):=\frac{e^{i k|z-\varsigma|}}{-4 \pi|z-\varsigma|}
$$

and

$$
\tilde{g}(z)=g(z)-2 \int_{\tilde{\Gamma}} g(\varsigma) \frac{\partial}{\partial n_{z}} \Phi(z, \varsigma) d s_{\varsigma}
$$

(ii) There exists exactly one solution $u \in H^{1 / 2}(\tilde{\Gamma})$ of (4.24).

(iii) Let $g \in H^{s}(\tilde{\Gamma}), s \geq-1 / 2$, and $\tilde{\Gamma}$ be $C^{\infty}$. Then the solution $u$ of (4.24.) belongs to $H^{s+1}(\tilde{\Gamma})$.

Correspondingly, using the direct approach of [20], [22], one obtains for the Dirichlet problem (4.19), (4.21), (4.22):

THEOREM 4.6. Let $f \in H^{1 / 2}(\tilde{\Gamma})$ be given. Then, with $k$ as above, there holds: (i) $u \in H_{\text {loc }}^{1}\left(\mathbf{R}^{3} \backslash \bar{\Omega}\right)$ solves (4.19), (4.21), (4.22) if and only if $\partial u / \partial n \in H^{-1 / 2}(\tilde{\Gamma})$ satisfies the integral equation

$$
V \frac{\partial u}{\partial n}(z):=-2 \int_{\tilde{\Gamma}} \frac{\partial u}{\partial n}(\varsigma) \Phi(z, \varsigma) d s_{\varsigma}=\tilde{f}(z), \quad z \in \Gamma,
$$

with $\Phi$ as in (4.25) and

$$
\tilde{f}(z):=f(z)+2 \int_{\tilde{\Gamma}} u(\varsigma) \frac{\partial}{\partial n_{\zeta}} \Phi(z, \varsigma) d s_{\zeta} .
$$

(ii) There exists exactly one solution $\partial u / \partial n \in H^{-1 / 2}(\tilde{\Gamma})$ of (4.27). 
(iii) Let $f \in H^{s}(\tilde{\Gamma}), s \geq 1 / 2$, and $\tilde{\Gamma}$ be $C^{\infty}$. Then the solution $\partial u / \partial n$ of (4.27) belongs to $H^{s-1}(\tilde{\Gamma})$.

Proofs of Theorems 4.5 and 4.6. For brevity we sketch only the main steps. The equivalence (i) between the boundary value problems and the integral equations is standard and follows immediately from Green's formula (see [16], [20]). The existence and uniqueness results (ii) in Theorems 4.5 and 4.6 are based on the strong ellipticity of the pseudodifferential operators $D$ and $V$, i.e., with constants $\tilde{\gamma}_{1}, \tilde{\gamma}_{2}>0$ and compact mappings $\tilde{C}_{1}: H^{1 / 2}(\tilde{\Gamma}) \rightarrow H^{-1 / 2}(\tilde{\Gamma})$ and $\tilde{C}_{2}: H^{-1 / 2}(\tilde{\Gamma}) \rightarrow$ $H^{1 / 2}(\tilde{\Gamma})$, there hold the Garding inequalities

$$
\begin{aligned}
& \operatorname{Re}\left\langle\left(D+\tilde{C}_{1}\right) v, v\right\rangle_{L^{2}(\tilde{\Gamma})} \geq \tilde{\gamma}_{1}\|v\|_{H^{1 / 2}(\tilde{\Gamma})}^{2} \\
& \operatorname{Re}\left\langle\left(V+\tilde{C}_{2}\right) \psi, \psi\right\rangle_{L^{2}(\tilde{\Gamma})} \geq \tilde{\gamma}_{2}\|\psi\|_{H^{-1 / 2}(\tilde{\Gamma})}^{2}
\end{aligned}
$$

for all $v \in H^{1 / 2}(\tilde{\Gamma})$ and $\psi \in H^{-1 / 2}(\tilde{\Gamma})$. Hence, $D: H^{1 / 2}(\tilde{\Gamma}) \rightarrow H^{-1 / 2}(\tilde{\Gamma})$ and $V: H^{-1 / 2}(\tilde{\Gamma}) \rightarrow H^{1 / 2}(\tilde{\Gamma})$ are Fredholm operators of index zero. Under the assumption (4.23) we have for $k \neq 0, \operatorname{Im} k \geq 0$, that the integral equations have no eigensolutions. Hence, the above mappings $D$ and $V$ are bijective, yielding assertion (ii). The regularity results (iii) in Theorems 4.5 and 4.6 follow in a standard way from the ellipticity of the pseudodifferential operators $D$ and $V$ (see for example [19], [20]).

Finally, we consider the Galerkin equations for the integral equations (4.24) and (4.27) and show the convergence of the $p$-version.

The Galerkin method ( $p$-version) for the integral equation (4.24) reads, with $V_{p}(\tilde{\Gamma})$ defined by $(2.7)$ :

Find $v_{p} \in V_{p}(\tilde{\Gamma})$ such that, with $\tilde{g} \in H^{-1 / 2}(\tilde{\Gamma})$ given by (4.26), there holds for all $\phi_{p} \in V_{p}(\tilde{\Gamma})$

$$
\left\langle D v_{p}, \phi_{p}\right\rangle_{L^{2}(\tilde{\Gamma})}=\left\langle\tilde{g}, \phi_{p}\right\rangle_{L^{2}(\tilde{\Gamma})} .
$$

Correspondingly, the Galerkin method (p-version) for the integral equation (4.27) reads, with $S_{p}(\tilde{\Gamma})$ given by $(2.6)$ :

Find $\psi_{p} \in S_{p}(\tilde{\Gamma})$ such that, with $\tilde{f} \in H^{1 / 2}(\tilde{\Gamma})$ given by (4.28), there holds for all $\phi_{p} \in S_{p}(\tilde{\Gamma})$

$$
\left\langle V \psi_{p}, \phi_{p}\right\rangle_{L^{2}(\tilde{\Gamma})}=\left\langle\tilde{f}, \phi_{p}\right\rangle_{L^{2}(\tilde{\Gamma})}
$$

THEOREM 4.7. Let $p$ be sufficiently large and $g \in H^{s}(\tilde{\Gamma}), s \geq-1 / 2$. Then the Galerkin equations (4.31) are uniquely solvable. Let $u \in H^{s+1}(\tilde{\Gamma})$ be the exact solution of (4.24) and $v_{p} \in V_{p}(\tilde{\Gamma})$ be the Galerkin solution. Then we have for $s \geq-1 / 2$

$$
\left\|u-v_{p}\right\|_{H^{1 / 2}(\tilde{\Gamma})} \leq C p^{-(s+1 / 2)}\|g\|_{H^{s}(\tilde{\Gamma})}
$$

with a constant $C$ independent of $u, \tilde{g}$ and $p$.

Proof. Obviously, since Gårding's inequality (4.29) holds, the assumptions of Lemma 4.1 are satisfied if we choose $A=D, H=H^{1 / 2}(\tilde{\Gamma}), H^{\prime}=H^{-1 / 2}(\tilde{\Gamma})$ and $S_{N}=V_{p}(\tilde{\Gamma}) \subset H^{1 / 2}(\tilde{\Gamma})$. Note that $\tilde{\Gamma}$ is a closed, bounded, $C^{\infty}$ surface. Thus, 
for $p$ large enough, Lemma 4.1 guarantees the unique solvability of the Galerkin equations (4.31) and the quasioptimal estimate for the Galerkin error

$$
\left\|u-v_{p}\right\|_{H^{1 / 2}(\tilde{\Gamma})} \leq C \inf \left\{\left\|u-w_{p}\right\|_{H^{1 / 2}(\tilde{\Gamma})}: w_{p} \in V_{p}(\tilde{\Gamma})\right\} .
$$

From Theorem 4.5 (iii) we know that for $s \geq-1 / 2$, with a constant $C$,

$$
\|u\|_{H^{s+1}(\tilde{\Gamma})} \leq C\|g\|_{H^{s}(\tilde{\Gamma})} .
$$

Therefore, we can apply the approximation result (3.1) of Theorem 3.1 to (4.34) and obtain (4.33) by using (4.35).

THEOREM 4.8. Let $p$ be sufficiently large and $f \in H^{s}(\tilde{\Gamma}), s \geq 1$. Then the Galerkin equations (4.32) are uniquely solvable. Let $\partial u / \partial n \in H^{s-1}(\tilde{\Gamma})$ be the exact solution of (4.27) and $\psi_{p} \in S_{p}(\tilde{\Gamma})$ be the Galerkin solution. Then we have for $s \geq 1$

$$
\left\|\frac{\partial u}{\partial n}-\psi_{p}\right\|_{H^{-1 / 2}(\tilde{\Gamma})} \leq C p^{-(s-1 / 2)}\|f\|_{H^{s}(\tilde{\Gamma})}
$$

with a constant $C$ independent of $\partial u / \partial n, \tilde{f}$ and $p$.

Proof. Again, application of Lemma 4.1 gives the assertion if we take $A=V$, $H=H^{-1 / 2}(\tilde{\Gamma}), H^{\prime}=H^{1 / 2}(\tilde{\Gamma})$ and $S_{N}=S_{p}(\tilde{\Gamma}) \subset H^{-1 / 2}(\tilde{\Gamma})$, since the Gårding inequality (4.30) holds. Note again that $\tilde{\Gamma}$ is a closed, bounded, $C^{\infty}$ surface. From Theorem 4.6 (iii) we know that for $s \geq 1$, with a constant $C$,

$$
\left\|\frac{\partial u}{\partial n}\right\|_{H^{s-1}(\tilde{\Gamma})} \leq C\|f\|_{H^{s}(\tilde{\Gamma})} .
$$

On the other hand, Lemma 4.1 yields

$$
\left\|\frac{\partial u}{\partial n}-\psi_{p}\right\|_{H^{-1 / 2}(\tilde{\Gamma})} \leq C \inf \left\{\left\|\frac{\partial u}{\partial n}-\phi_{p}\right\|_{H^{-1 / 2}(\tilde{\Gamma})}: \phi \in S_{p}(\tilde{\Gamma})\right\} .
$$

Therefore, by applying Theorem 3.2 and Remark 3.1 to (4.38) we obtain with (4.37) the desired estimate (4.36).

Remark 4.2. Theorems 4.7, 4.8 show that for the $p$-version, the rate of convergence obtained depends only upon the smoothness of the data. Hence, when $\tilde{f}$ and $\tilde{g}$ are arbitrarily smooth, one obtains arbitrarily high rates of convergence. This is in direct contrast to the $h$-version, where the rate of convergence depends in addition upon the degree of polynomials used, and is therefore not very high, even for smooth solutions.

Finally, we remark that results analogous to those above can be shown for twodimensional crack problems in linear elasticity, since these problems can be reduced to first-kind integral equations like (4.6) or (4.14) for the components of the jumps of the displacement or traction across the crack line $\Gamma$. Regularity results analogous to (4.10) and (4.15) hold for the solutions (see [17], [22], [23]). Hence, the Galerkin schemes corresponding to (4.11) and (4.17) will lead componentwise to error estimates like (4.12) and (4.18) with obvious modifications.

Acknowledgments. The first author was supported by the National Science Foundation under Grant DMS-8603954. The second author was supported by Air 
Force Office of Scientific Research, Air Force Systems Command, USAF, under Grant number AFOSR-85-0322.

School of Mathematics

Georgia Institute of Technology

Atlanta, Georgia 30332

Department of Mathematics and Statistics

University of Maryland, Baltimore County

Catonsville, Maryland 21228

1. E. Alarcon, L. Abia \& A. Reverter, "On the possibility of adaptive boundary elements," in Accuracy Estimates and Adaptive Refinements in Finite Element Computations (AFREC), Lisbon, 1984.

2. E. Alarcon, A. Reverter \& J. Molina, "Hierarchical boundary elements," Comput. \& Structures, v. 20, 1985, pp. 151-156.

3. E. AlarCon \& A. REverter, "p-adaptive boundary elements," Internat. J. Numer. Methods Engrg., v. 23, 1986, pp. 801-829.

4. I. BABUŠKA, The $p$ and h-p Versions of the Finite Element Method. The State of the Art, Technical Note BN-1156, Institute for Physical Science and Technology, University of Maryland, College Park, MD, 1986.

5. I. BABUSKKA, B. A. SZABO \& I. N. KATZ, "The p-version of the finite element method," SIAM J. Numer. Anal., v. 18, 1981, pp. 515-545.

6. I. BABUŠKA \& M. SURI, The Optimal Convergence Rate of the $p$-Version of the Finite Element Method, Technical Note BN-1045, Institute for Physical Science and Technology, University of Maryland, College Park, MD, 1985.

7. I. BABUŠKA \& M. SURI, The Treatment of Nonhomogeneous Dirichlet Boundary Conditions by the $p$-Version of the Finite Element Method, Institute for Physical Science and Technology, University of Maryland, College Park, MD, 1987.

8. I. BABUŠKA \& M. SURI, "The $h$ - $p$ version of the finite element method with quasiuniform meshes," Math. Modeling Numer. Anal. RAIRO, v. 21, 1987, pp. 199-238.

9. I. BABUŠKA \& M. SURI, "The $p$-version of the finite element method for constraint boundary conditions," Institute for Physical Science and Technology, University of Maryland, College Park, MD, 1987.

10. C. A. Brebbia, Editor, Progress in Boundary Element Methods, Vols. 1, 2, 3, 4, 5, SpringerVerlag, Berlin, $1981 \mathrm{ff}$.

11. M. COSTABel \& E. P. STEPhAN, "Boundary integral equations for mixed boundary value problems in polygonal domains and Galerkin approximations," in Mathematical Models and Methods in Mechanics 1981 (W. Fiszdon and K. Wilmánski, eds.), Banach Center Publications Vol. 15, PWN-Polish Scientific Publishers, Warsaw 1985, pp. 175-251.

12. M. R. DORR, "The approximation theory for the $p$-version of the finite element method," SIAM J. Numer. Anal., v. 21, 1984, pp. 1180-1207.

13. W. GUI \& I. BABUŠKA, "The $h, p$ and $h-p$ versions of the finite element method in one dimension," Parts 1-3, Numer. Math., v. 49, 1986, pp. 577-683.

14. B. GuO \& I. BABUŠKA, "The $h-p$ version of the finite element method. Part 1: The basic approximation results," Comput. Mech., v. 1, 1986, pp. 21-41; "Part 2: General results and applications," Comput. Mech., v. 1, 1986, pp. 203-220.

15. S. HiLdebrandT \& E. WiEnhOlTZ, "Constructive proofs of representation theorems in separable Hilbert space," Comm. Pure Appl. Math., v. 17, 1964, pp. 369-373.

16. G. C. HSIAO \& W. L. WENDLAND, "A finite element method for some integral equations of the first kind," J. Math. Anal. Appl., v. 58, 1977, pp. 449-481.

17. G. C. HSiAO, E. P. STEPhAN \& W. L. WENDland, An Integral Equation Formulation for a Boundary Value Problem of Elasticity in the Domain Exterior to an Arc, Lecture Notes in Math., vol. 1121, 1983, pp. 153-165.

18. J. L. Lions \& E. MAGenes, Non-Homogeneous Boundary Value Problems and Applications. I, Springer-Verlag, Berlin and New York, 1972.

19. B. E. Petersen, Introduction to the Fourier Transform and Pseudodifferential Operators, Pitman, Boston, 1983. 
20. E. P. STEPhan, Boundary Integral Equations for Mixed Boundary Value Problems, Screen and Transmission Problems in $\mathbf{R}^{3}$, Habilitationsschrift, Technische Hochschule Darmstadt, 1984.

21. E. P. STEPHAN \& W. L. WENDLAND, "Remarks to Galerkin and least squares methods with finite elements for general elliptic problems," Manuscripta Geodaetica, v. 1, 1976, pp. 93-123.

22. E. P. STEPHAN \& W. L. WENDLAND, "An augmented Galerkin procedure for the boundary integral method applied to two-dimensional screen and crack problems," Appl. Anal., v. 18, 1984, pp. 183-219.

23. E. P. STEPhaN \& W. L. WENDland, "A hypersingular boundary integral method for two-dimensional screen and crack problems," Arch. Rational Mech., 1989. (To appear.)

24. W. L. WENDLAND, "Boundary element methods and their asymptotic convergence," in Theoretical Acoustics and Numerical Techniques (P. Filippi, ed.), CISM Courses 277, Springer-Verlag, Wien, New York, 1983, pp. 135-216.

25. W. L. WENDLAND, "On some mathematical aspects of boundary element methods for elliptic problems," in Mathematics of Finite Elements and Applications V (J. Whiteman, ed.), Academic Press, London, 1985, pp. 193-227.

26. W. L. WENDLAND, "Splines versus trigonometric polynomials-the $h$-versus the $p$-version in two-dimensional boundary integral methods," in Dundee Biennial Conference on Numerical Analysis (D. F. Griffiths and G. A. Watson, eds.), pp. 238-255. Longman Scientific \& Technical, Harlow, Essex, 1986. 